\title{
Identification of Company-Specific Stress Scenarios in Non-Life Insurance
}

\section{Wiltrud Weidner, J.-Matthias Graf von der Schulenburg}

Institute for Risk and Insurance, Leibniz University Hanover, Hanover, Germany

Email address:

ww@ivbl.uni-hannover.de (W. Weidner),jms@ivbl.uni-hannover.de (J.-Matthias G. von der Schulenburg)

\section{To cite this article:}

Wiltrud Weidner, J.-Matthias Graf von der Schulenburg. Identification of Company-Specific Stress Scenarios in Non-Life Insurance. Applied and Computational Mathematics. Special Issue: Computational Methods in Monetary and Financial Economics.

Vol. 5, No. 1-1, 2016, pp. 1-13. doi: 10.11648/j.acm.s.2016050101.11

\begin{abstract}
This paper provides an effective approach, known as dynamic financial analysis, to the systematic development of stress scenarios for the risk profile of non-life insurers, which can be used in risk analysis for the regulatory and rating assessment. The determination of company-specific stress scenarios is demonstrated, the resulting critical scenarios are described. Non-linear dependencies have a significant impact on the scenarios, some of which have not previously been adequately considered are introduced. The recent global financial crisis illustrates that the analysis of extreme events, which can affect both sides of the balance sheet, is essential in an asset-liability management context.
\end{abstract}

Keywords: Non-Life Insurance, Solvency II, Risk Management, Dynamic Financial Analysis, Stress Testing, Copulas

\section{Introduction}

In a deregulated insurance market, in which stateprescribed large margins do not replace risk awareness, stock price jumps, valuation changes of entire asset classes and major loss scenarios caused by natural disasters or technical innovations necessitate the subsequent inclusion of extreme scenarios into the business plan. Under the EU insurance law, Solvency II, the development of reliable stress test procedures is now required. Even the International Association of Insurance Supervisors (IAIS) has mentioned the possible company-specific development of stress tests related to the functioning and usefulness. Stress tests used to explore especially unfavorable trends are an indispensable element of risk management in insurance companies [1]. In practice, such stress tests constitute an important addition to the stochastic methods based on an economic view and to the risk-based-capital concepts with a static character, as currently exercised in the USA, Canada and Australia. Development scenarios in stress tests are assumed to be deterministic. They are used to verify their impact on the balance sheet and typically consider scenarios involving extreme and therefore rare developments in the capital market; examples include the German 'Stresstest' of the Bundesanstalt für Finanzdienstleistungsaufsicht (BaFin) [2], the 'Resilience Test for Life Insurers' and the 'Reverse-Stress
Test' of the Financial Services Authority (FSA) in the United Kingdom [3]. The background of these aforementioned scenarios are financial theoretical considerations based on historical data. In the banking sector, stress testing using such hand-picked pure scenarios also originated in the evaluation of market risk [4] and has been extended to credit risk [5] and macro analysis [6]. Stress scenarios focusing on the company-specific risk situation of an insurer can also be derived using an entirely different approach known as dynamic financial analysis (DFA) [7]. Non-linear dependencies are particularly important in the modeling of extreme events [8].

DFA is a tool for the systematic and holistic analysis of a company's financial performance for several time periods into the future [9]. In this paper, the influence of extreme events on a non-life insurer is examined based on a companyspecific DFA model that reflects the risk and return profile of the company, considering the mutual dependence of the key risk drivers. Non-linear dependencies are modeled using various types of copulas, whose effects are studied in the context of stress testing. A practical and flexible approach to the determination of stress scenarios is presented, in which a cluster analysis based on selected worst scenarios is performed. 
An important outcome of this study is that non-linear dependencies and the number of considered critical scenarios have a strong influence on the form and composition of the sources of risk. This result should be taken into account by the supervisory authorities and rating agencies. For example, a very unfavorable development in the number and amount of claims could occur together with a very unfavorable capital market trend at the same time. Therefore, it is clearly insufficient to consider only selected capital market scenarios in risk management.

The current paper focuses on two themes: the application of DFA and the integration of copulas into them. DFA became an important financial analysis tool in insurance undertakings in the late 1990s. The Casualty Actuarial Society (CAS) made a decisive contribution to the field through its abundance of background materials [9]. Meantime, there are numerous treatises in the scientific literature. The basic DFA structure was presented by [10] and [11], along with example applications. In [12-14], DFA was applied as a decision-making tool. The theoretical foundation of the copula concept was introduced by [15]. However, copulas were only established into the mathematics of insurance in the late 1990s by [16] and [17]. An overview of the applications of copulas to financial mathematics was provided by [18]. In [19-21], various problems on copula estimation were studied. Various algorithms for constructing Archimedean copulas were presented in [22-24].

The current analysis builds directly on a DFA model presented in [25]. In this model, various types of copulas are implemented, and the effects of the employed copulas on the gain and risk of an insurer are analyzed. In [25], large differences in the risk measures depending on the copula concept are found. However, certain aspects of DFA implementation have not been analyzed in the previous literature, despite the growing importance of the DFA framework in theory and practice. One aspect that has not been considered is the integration of stress testing into DFA models. The aim of the present study is to identify stress scenarios for non-life insurers under different dependence structures, which can then be used for stress testing. In contrast to the traditional definition, a stress scenario is defined in this paper by negative economic attributes in all of the underlying risk factors. Not only are the impacts of the scenarios on the investments modeled, but also their impacts on the other items in the balance sheet. A fundamental finding of this paper is that it is crucial to consider non-linear dependencies to improve the determination of stress scenarios. This result is important from both the regulatory perspective and the rating agency's as well, as traditional stress scenarios can underestimate the company-specific risk. Since stress tests are essential in risk management, appropriate stress scenarios derived from a DFA model should be incorporated into the regulatory and rating assessment frameworks.

The remainder of the article is organized as follows. In Section 2, the concept of stress testing based on DFA is introduced. The method for constructing stress scenarios using a DFA model is presented in Section 3. In Section 4, the derivation of extremely severe scenarios, including the mathematical modeling techniques and implementation of non-linear dependencies, is demonstrated through a simulation study. Section 5 concludes the paper.

\section{Integration of Stress Testing in DFA}

The DFA model oriented to the structure of, e.g., [11] and [26] has been created and developed to simulate the evolution of the financial and risk situation of an insurer for a wide range of possible scenarios. 1 The simulation results demonstrate how internal and external conditions can influence the financial results of the company [9]. The DFA definition of the CAS (see [9], Chapter 6, p. 2 et seq.) states that "[...] The process of DFA involves testing a number of adverse and favorable scenarios regarding an insurance company's operations. DFA assesses the reaction of the company's surplus to the various selected scenarios." The strength of the DFA model lies precisely in the fact that in its run, individual adverse scenarios are implicitly analyzed as well as individual favorable scenarios. This approach is similar that of stress testing [1]. Additional facts are included by the DFA that are not considered in other models representing only 'normal' business performance.

However, the term 'scenario' is not clearly defined. More precisely, we must distinguish linguistically between a scenario that is an assumption in a simulation and a scenario that is a result of a simulation; note that the input variables of a stochastic model are related to its output, because some variables must be modified during the simulation. The CAS differentiates between two mutually exclusive definitions of scenarios in a stochastic model [9]: the 'parameter scenario' and the 'run scenario'. The parameter scenario can be described as the summary of the employed assumptions regarding the underlying distributions. For example, catastrophic losses can be modeled by a Pareto distribution with a specified mean and dispersion parameter. Whereas the run scenario represents an individual run of the parametric model. For example, if the parametric model runs 500,000 times, and therefore 500,000 simulations are taken into account for the analysis, each of the 500,000 runs can be considered as a scenario.

\section{Implementation of Stress Testing in a DFA Framework}

This section focuses on the determination and evaluation of adverse scenarios based on DFA simulation results. Therefore, the term 'scenario' is understood to refer to a run scenario presented in the previous section.

For identification of adverse scenarios the proportionate

1 The DFA is based on a stochastic model, e.g. the future financial and risk situation in the non-life insurance industry is measured by means of an extensive stochastic modeling as opposed to traditional scenario analysis with selected deterministic scenarios [11] 
worst scenarios among the generated simulations, i.e., those yielding the most extreme financial results over the observed time horizon, can be investigated. High demands are therefore made on the input and the model framework. In particular, parameter values that lead to poor results have been admitted. For example, the company's business result may be affected by the selected height of the volatility of the investment return. In the framework, the algorithms must exclude impossible results; otherwise, the algorithms must not unnecessarily restrict the range. The quality of the DFA model depends on its functionality within the boundary area. For extreme results, cases in which the company earns an exorbitant profit are less interesting; results leading to the company's ruin and disappearance from the market are more interesting, leading to the term 'poor results'.

In this paper, the term 'poor' is used to describe those scenarios that lead to the company's economic ruin during the selected time horizon. The classification for poor scenarios is based on two criteria. The sooner the ruin occurs, the more unfavorable the result of the simulation is perceived, thus the first criterion is the time of the ruin. The next criterion for classification is the amount of the loss. The more negative the equity capital is, the worse the result of the simulation. All of the results can be standardized for better comparison. The scaling can be performed using discounting by the riskfree rate of return at time 0 to achieve a uniform view.

The risk factors of the scenarios which lead to poor results normally show a wide range of characteristics. To account for data with different types of attributes, it is appropriate to group them into classes to allow a better analysis based on the obtained structuring. The cluster analysis can be used for the classification. 2 In clustering, the data are summarized based on their similarity. Objects within the same cluster have strong similarities to one another in terms of their characteristics but are dissimilar to objects in other clusters [27].

A number of different methods have been developed for the identification of clusters in [28-31]. In the following simulation study, the clusters are identified using the Ward's minimum variance method [32], implemented recursively using the Lance-Williams algorithm [33].3 The partition can be determined by considering large jumps in the increase of the heterogeneity. Ward's method is an agglomerative hierarchical clustering procedure in which groups are gradually merged. In practice, the method generates highly homogenous, compact groups and constitutes a stable procedure. It is one of the most widely used methods in clustering analysis [34].

In the approach taken in this paper, the clusters are defined

2 The risk factors in the poor scenarios have different characteristics, arguing against using an average observation, which would lead to the loss of too much relevant information. More information is maintained by considering quantiles. However, it is still impossible to extract the relations between the risk factors.

3 Applying Ward's method appears to be plausible because it is directly related to the Euclidean distance. The poor scenarios with, e.g., similar investment returns and loss ratios have a Euclidean distance close to zero. They are pooled into one group, and the within-group sum of squares is small. to be stress scenarios. For this purpose, the classification of poor scenarios should be chosen in such a way that risk factors and financial values within a given cluster are comparable. The objective of reducing the variety of individual cases in a given group to one case corresponding to the mean of the individual cases can thereby be achieved. The obtained stress scenarios are specific to the profile of the insurer and are therefore realistic and objective despite their severity. In a stress test using these adverse scenarios, all of the risk factors that affect the solvency of an insurance company over a prescribed time period are included. Stress testing using such mixed scenarios addresses all of the relevant risk categories that are reflected in the DFA model.

\section{Stress Scenarios in a Simulation Study}

\subsection{Simulation Model}

The analysis is intended to focus on the general method of identifying stress scenarios. A simplified model of a non-life insurance company using real-world data from a German insurer based on [14] and [25] is therefore employed. Tables 5-8 in the appendix summarize the model assumptions and parameters.

The simulation study is performed for a five-year time period. Two types of liabilities are differentiated in the loss model, non-catastrophic losses and catastrophic losses. The underwriting result at the end of period $t$, which assesses the development of the accident year exactly, is applied as a measure of the underwriting profitability. This quantity consists of the premiums less the claims and expenses (upfront and claims settlement costs):

$$
U_{t}=P_{t-1}-C_{t}-E x_{t-1}^{P}-E x_{t}^{C}, t=1,2, \ldots, 5 .
$$

On the investment side, a distinction is made between high-risk investments (with portion $\alpha_{t-1}$ and return $r_{1 t}$ in period $t$ ), such as stocks, and low-risk investments (with portion $\left(1-\alpha_{t-1}\right)$ and return $r_{2 t}$ in period $\left.t\right)$, such as government bonds. The investment result at the end of period $t$ is calculated by multiplying the portfolio return by the free capital:

$$
\begin{gathered}
I_{t}=r_{p t} \cdot A_{t-1} \text { with } r_{p t}=\alpha_{t-1} \cdot r_{1 \mathrm{t}}+\left(1-\alpha_{t-1}\right) \cdot r_{2 \mathrm{t}}, t=1, \\
2, \ldots, 5 .
\end{gathered}
$$

given a tax rate $t r$ on positive earnings, the company's earning is computed from the underwriting and investment result as follows:

$$
E_{t}=I_{t}+U_{t}-\max \left(t r \cdot\left(I_{t}+U_{t}\right), 0\right), t=1,2, \ldots, 5 .
$$

Finally, the development of the equity capital at the end of period $t$ is described as follows:

$$
E C_{t}=E C_{t-1}+E_{t}, t=1,2, \ldots, 5 .
$$

The model depicts two asset classes, high-risk and lowrisk investments, and two types of liabilities, noncatastrophic and catastrophic losses. In modeling the 
dependencies between these risk categories, correlated random numbers are generated. The measurement of the dependencies is described via the rank correlation coefficient Kendall's tau [35], which captures non-linear dependencies, in contrast to the commonly used linear correlation coefficient. 4 The correlations are modeled using various copula types from two important copula families: elliptical copulas (Gauss, t) and Archimedean copulas (Gumbel, Clayton, Frank). 5 These five copulas, including their survival copulas, differ in the form of their tail dependence; the properties of the copulas are summarized in Table 9 and 10 in the appendix [18,37].6 They are suitable for stress scenario analysis due to the different effects of the different forms of tail dependence [25]. In addition, they are widely applied in actuarial practice and their fitting to data is quite simple [39].

Typical management strategies, e.g., growth or solvency strategies, often fail in extreme scenarios because they are designed for the normal development of the insurance company [40]. It therefore appears sufficient to assume that the model parameters are unaffected by the management rules in the analysis of stress scenarios.

In the analysis of the financial and risk situation, eight different measurements of the risk, return and performance are calculated. The expected gain per period is used as an absolute measure of return:

$$
E(G)=\frac{E\left(E C_{5}\right)-E C_{0}}{5} .
$$

The return on investment, based on the ratio of the expected gain to the invested capital, is used as a relative measure of return:

$$
R O I=\left(\frac{E\left(E C_{5}\right)}{E C_{0}}\right)^{\frac{1}{5}}-1
$$

A distinction is made between measures of the total risk and downside risk, and measures describing the risk as a risk capital requirement are also distinguished. The standard deviation of the gain per period is a total risk measure because it measures the deviation from the expected value in two directions:

4 Concretely, three dependencies on two levels are represented in the model: The investment returns are correlated through the parameter $\rho_{\tau 1}$. The non-catastrophic losses are connected to the catastrophic losses through the parameter $\rho_{\tau 2}$ and the assets are connected to the liabilities through the parameter $\rho_{\tau 3}$.

5 The three Archimedean copulas and their respective survival copulas are implemented as hierarchical Archimedean copulas (HACs) [22,36]. HACs are a generalization of multivariate Archimedean copulas, with improve flexibility, as they do not depend on a single parameter. In contrast to normal Archimedean copulas, HACs define the dependence structure recursively. In the fourdimensional case, the copula function is given by $C\left(u_{1}, u_{2}, u_{3}, u_{4}\right)=\varphi_{3}\left(\varphi_{3}^{-1}\right.$ 。 $\left.\varphi_{1}\left(\varphi_{1}^{-1}\left(u_{1}\right)+\varphi_{1}^{-1}\left(u_{2}\right)\right)+\varphi_{3}^{-1} \circ \varphi_{2}\left(\varphi_{2}^{-1}\left(u_{3}\right)+\varphi_{2}^{-1}\left(u_{4}\right)\right)\right)$, with three generator functions $\varphi_{1}, \varphi_{2}$ and $\varphi_{3}$.

6 Tail dependence is defined as follows in [38]: $\lambda_{l}:=\lim _{u \downarrow 0} P\left(X_{2} \leq F_{X_{2}}^{-1}(u) \mid X_{1} \leq\right.$ $\left.F_{X_{1}}^{-1}(u)\right)$ is the lower tail dependence coefficient and $\lambda_{u}:=\lim _{u \uparrow 1} P\left(X_{2}>F_{X_{2}}^{-1}(u)\right.$ । $\left.X_{1}>F_{X_{1}}^{-1}(u)\right)$ is the upper tail dependence coefficient for two random variables $X_{1}$ and $X_{2}$ with marginal distributions $F_{X_{1}}$ and $F_{X_{2}}$. A positive coefficient $\lambda_{u}\left(\lambda_{l}\right)$ shows that the two random variables are asymptotically dependent in the upper (lower) tail; a vanishing coefficient indicates that they are asymptotically independent.

$$
\sigma(G)=\frac{\sigma\left(E C_{5}\right)}{5}
$$

In contrast, downside risk measures, such as the ruin probability or expected policyholder deficit, take only negative deviations into account. The ruin probability can be obtained as follows:

$$
R P=\operatorname{Pr}(\hat{\tau} \leq 5), \text { with } \hat{\tau}=\inf \left\{t>0 \mid E C_{t}<0, t=\right.
$$

where $\hat{\tau}$ indicates the date of the first occurrence of ruin [41]. Information on the amount of loss in the event of insolvency is available through the expected policyholder deficit:

$$
E P D=\sum_{t=1}^{5} E\left(\max \left(-E C_{t}, 0\right) \mid E C_{t}<0\right) \cdot\left(1+r_{f}\right)^{-t},(9)
$$

where $r_{f}$ is the risk-free rate of return. The risk capital can be determined from risk measures such as the value at risk and the tail value at risk. A loss $L_{t}=-\left(E C_{t}-E C_{t-1}\right)$, for period $t=1,2, \ldots, 5$ with distribution function $F_{L_{t}}$, is therefore assumed. Assuming a significance level $0<\alpha<1$, the value at risk is defined as the lower $(1-\alpha)$-quantile of $F_{L_{t}}$ [42-43]:

$$
\operatorname{VaR}_{\alpha}\left(L_{t}\right)=Q_{1-\alpha}\left(L_{t}\right)=\inf \left\{x \mid F_{L_{t}}(x) \geq 1-\alpha\right\},
$$

and the tail value at risk is defined as the expected loss of the $100 \cdot \alpha$ percent worst cases:

$$
\operatorname{TVaR}_{\alpha}\left(L_{t}\right)=E\left(L_{t} \mid L_{t} \geq \operatorname{VaR}_{\alpha}\left(L_{t}\right)\right) .
$$

Along these lines, a multi-year risk capital concept assuming a time horizon of five periods is considered [26]. The random variable MaxLoss, which corresponds to the maximum of the cumulative loss of each simulation, is defined as follows:

$$
\operatorname{Max} \operatorname{Loss}(T)=\max \left\{\operatorname{CumLoss}_{t} \mid t=1,2, \ldots, 5\right\},
$$

where CumLoss $_{1}=L_{1} \cdot\left(1+r_{f}\right)^{-1}$ and CumLoss $=$ CumLoss $_{t-1}+L_{t} \cdot\left(1+r_{f}\right)^{-t}, t=2, \ldots, 5$, with the riskfree rate of return $r_{f}$. Therefore, the insurance can cover all losses over the entire time horizon without further additional capital if this amount is available at $t=0$ in the simulation path. In the calculation of the multi-year risk capital requirement, selected risk measures, such as the $\operatorname{VaR}$ and $T V a R$, can be applied.

Furthermore, three performance measures are considered, each differing in the numerator. The Sharpe ratio, based on the standard deviation, is the ratio of the risk premium and the standard deviation of the returns [44]:

$$
S R_{\sigma}=\frac{E\left(E C_{T}\right)-E C_{0} \cdot\left(1+r_{f}\right)^{T}}{\sigma\left(E C_{T}\right)}
$$

This ratio measures both the negative and positive deviations of the returns in relation to the expected value. Both deviations are not captured if the ruin probability or the $E P D$ is used in place of the standard deviation:

$$
S R_{R P}=\frac{E\left(E C_{T}\right)-E C_{0} \cdot\left(1+r_{f}\right)^{T}}{R P}
$$




$$
S R_{E P D}=\frac{E\left(E C_{T}\right)-E C_{0} \cdot\left(1+r_{f}\right)^{T}}{E D P} .
$$

These two ratios include only negative deviations of the returns.

\subsection{Original Simulation Results}

The results for eight dependence structures generated from
500,000 simulations on the basis of a Monte Carlo simulation are shown in Table 1. The case assuming independence results in an expected gain $E(G)$ of $€ 28.56$ million, a return on investment $R O I$ of $23.76 \%$ and a standard deviation $\sigma(G)$ of $€ 15.44$ million per period. The ruin probability $R P$ is $0.35 \%$ for the five-period time horizon.

Table 1. Simulated return, risk and performance profile.

\begin{tabular}{lllllllll}
\hline Dependence Structure & No corr. & Gauss & t & Gumbel & Survival Gumbel & Clayton & Survival Clayton & Frank \\
\hline Tail Dependence & none & none & upper, lower & upper & lower & lower & upper & none \\
\hline $\mathrm{E}(\mathrm{G})$ in mill. $€$ & 28.56 & 28.04 & 27.98 & 28.09 & 27.80 & 27.70 & 28.16 & 28.08 \\
$\mathrm{ROI}$ & $23.76 \%$ & $23.47 \%$ & $23.43 \%$ & $23.49 \%$ & $23.33 \%$ & $23.27 \%$ & $23.54 \%$ & $23.49 \%$ \\
$\sigma(\mathrm{G})$ in mill. $€$ & 15.44 & 17.91 & 17.89 & 19.38 & 19.78 & 20.67 & 19.03 & 17.63 \\
$\mathrm{RP}$ & $0.35 \%$ & $1.16 \%$ & $1.40 \%$ & $0.89 \%$ & $1.90 \%$ & $1.91 \%$ & $0.81 \%$ & $0.93 \%$ \\
$\mathrm{EPD}$ in mill. $€$ & 0.04 & 0.15 & 0.21 & 0.93 & 1.40 & 1.95 & 0.83 & 0.13 \\
$\mathrm{VaR}_{0,005}$ (MaxLoss) in mill. $€$ & 230.54 & 293.39 & 326.89 & 270.99 & 361.69 & 354.67 & 258.42 \\
$\mathrm{TVaR}_{0,005}$ (MaxLoss) in mill. $€$ & 278.59 & 349.17 & 404.34 & 488.88 & 669.65 & 768.01 & 457.74 \\
$\mathrm{SR}_{\sigma}$ & 1.69 & 1.43 & 1.43 & 1.33 & 1.28 & 1.22 & 1.35 & 269.39 \\
$\mathrm{SR}_{\mathrm{RP}}$ & 36.94 & 11.04 & 9.16 & 14.41 & 6.68 & 6.61 & 15.99 & 1.46 \\
$\mathrm{SR}_{\mathrm{EPD}}$ & 3.18 & 0.83 & 0.61 & 0.14 & 0.09 & 0.06 & 0.15 & 13.75 \\
\hline
\end{tabular}

$\mathrm{E}(\mathrm{G})$ : expected gain per period; ROI: return on investment; $\sigma(\mathrm{G})$ : standard deviation of the gain per period; RP: ruin probability; EPD: expected policyholder deficit; $\mathrm{VaR}_{0.005}$ (MaxLoss): five-year risk capital using the $99.5 \%$ VaR; $\mathrm{TVaR}_{0.005}$ (MaxLoss): five-year risk capital using the $99.5 \% \mathrm{TVaR} ; \mathrm{SR}_{\sigma}$ : Sharpe ratio based on standard deviation; $\mathrm{SR}_{\mathrm{RP}}$ : Sharpe ratio based on ruin probability; $\mathrm{SR}_{\mathrm{EPD}}$ : Sharpe ratio based on expected policyholder deficit.

The comparison with the different dependence structures illustrates that the impact of the dependence structure on the expected gain is relatively limited. The expectation $E(G)$ is reduced by $1.39 \%$ to $3.02 \%$, depending on the copula type. For example, using the Frank copula (in which only linear dependencies are taken into account), the expected gain is reduced by $1.68 \%$, from $€ 28.56$ million to $€ 28.08$ million per period. The picture is similar for the return on investment. However, the correlation assumptions have a strong influence on the considered risk measures. Depending on the copula type, the standard deviation $\sigma(\mathrm{G})$ increases by between $14.15 \%$ (from $€ 15.44$ million to $€ 17.63$ million using the Frank copula) and $33.86 \%$ (from $€ 15.44$ million to $€ 20.67$ million using the Clayton copula). The five-year risk capital using the downside risk measure value at risk (VaR), which is $€ 230.54$ million assuming independence, increases by between $12.09 \%$ (to $€ 258.42$ million using the survival Clayton copula) and $56.88 \%$ (to $€ 361.69$ million using the survival Gumbel copula). The five-year risk capital using the downside risk measure tail value at risk (TVaR) grows faster for some copulas because of the 'I-point-consideration' property of the VaR. The risk capital increases by between $15.38 \%$ (from $€ 278.59$ million to $€ 321.43$ million using the Frank copula) and $175.65 \%$ (from $€ 278.59$ million to $€ 768.01$ million using the Clayton copula). Even greater changes occur in the other two downside risk measures, the ruin probability RP and the expected policyholder deficit EPD. Using the Gauss copula, the RP increases from $0.35 \%$ to $1.16 \%(+228.06 \%)$ and the EPD increases from $€ 0.04$ million to $€ 0.15$ million ( $+273.72 \%)$. As a consequence, the performance measured by using the Sharpe ratio (SR) is lower compared to the uncorrelated case: $\mathrm{SR} \sigma$ decreases by $15.48 \%$, SRRP decreases by $70.12 \%$ and $\mathrm{SR}_{\mathrm{EPD}}$ decreases by $73.77 \%$. The extreme effects are related to the type of dependency. The ruin probabilities are lower for copulas exhibiting dependence in the upper tail compared to the ruin probabilities for copulas that display no tail dependence; these probabilities are in turn lower than the ruin probabilities using copulas exhibiting lower tail dependence. The expected policyholder deficit and five-year risk capital using the TVaR are higher for the non-symmetric copulas than for the symmetric copulas. This stems from the fact that the non-symmetric copulas generate more extreme values in the tail compared to the symmetric copulas. The EPD and TVaR display both lower tail dependence and asymmetry of the copulas.

\subsection{Analysis of Poor Scenarios Using Clustering}

Considering the chosen dependence structure, a different number of scenarios may satisfy the definition of a poor scenario (see Table 2). The relations between the ruin probabilities for the various dependence structures are reflected here. For example, 1,771 scenarios $(0.35 \%$ of all runs) lead to the company's economic ruin over the considered time horizon of five periods for the case with no correlations, versus 9,371 scenarios ( $1.87 \%$ of all runs) for the case where the Clayton copula is used. The distribution of the scenarios across the single periods is quite similar for all of the dependence structures. In the first period, there are fewer poor scenarios than in the following years. 
Table 2. Number of poor scenarios.

\begin{tabular}{|c|c|c|c|c|c|c|c|c|}
\hline Dependence Structure & No corr. & Gauss & $\mathbf{t}$ & Gumbel & Survival Gumbel & Clayton & Survival Clayton & Frank \\
\hline Tail Dependence & none & none & upper, lower & upper & lower & lower & upper & none \\
\hline Period 1 & 63 & 333 & 978 & 178 & 1,555 & 1,352 & 106 & 144 \\
\hline Period 2 & 343 & 1,288 & 1,571 & 874 & 2,245 & 2,295 & 709 & 912 \\
\hline Period 3 & 470 & 1,893 & 1,704 & 1,144 & 2,225 & 2,271 & 1,050 & 1,241 \\
\hline Period 4 & 484 & 1,131 & 1,535 & 1,155 & 1,860 & 1,871 & 1,054 & 1,253 \\
\hline Period 5 & 411 & 1,165 & 1,192 & 1,024 & 1,519 & 1,582 & 958 & 1,062 \\
\hline Total & 1,771 & 5,810 & 6,980 & 4,375 & 9,404 & 9,371 & 3,877 & 4,612 \\
\hline
\end{tabular}

Examples of poor scenarios are the worst 15 scenarios and the corresponding sources of risk and financial results for the case with no correlation shown in Table 3. These scenarios already lead to the company's ruin in the first period $\left(\mathrm{EC}_{1}<\right.$ $0)$. The high-risk investment return is below $-25 \%$ on average, and the range of the return is between $-3 \%$ and $-50 \%$. The low-risk investment return is not nearly as unfavorable due to its lower volatility; the low-risk investment return is $0 \%$ on average. There are scenarios in which the low-risk investment return is close to the expected value of $5 \%$ as well as scenarios for which the return is nearly $-7 \%$. The loss ratio is quite high for each of the worst 15 scenarios. In most cases, the loss ratio is significantly above $100 \%$ (the expected value is $85.25 \%$ ) and varies by up to $30 \%$. The expense ratio is approximately $11 \%$. Given that the claims settlement costs depend on the claims through a percentage factor, so that a high loss ratio induces a high expense ratio, the expense ratios fluctuate. The wide range of characteristics of the specific risk factors has the effect that roughly equally high losses can stem from different causes. For instance, the loss can be affected equally by a negative underwriting and investment result (see the 11th worst scenario), a very high underwriting loss (see the 12th worst scenario) or a very negative investment result (see the 13th worst scenario).

Table 3. The worst 15 scenarios, assuming independence.

\begin{tabular}{|c|c|c|c|c|c|c|c|c|c|c|c|c|}
\hline & $\begin{array}{l}\mathrm{EC}_{0} \text { in } \\
\text { mill. } €\end{array}$ & $\begin{array}{l}\mathrm{EC}_{1} \text { in } \\
\text { mill. } €\end{array}$ & $\mathbf{r}_{11}$ & $\mathbf{r}_{21}$ & $\mathbf{r}_{\mathrm{p} 1}$ & $\begin{array}{l}C_{\text {ncat, } 1} \text { in } \\
\text { mill. } €\end{array}$ & $\begin{array}{l}\mathrm{C}_{\text {cat }, 1} \text { in } \\
\text { mill. } €\end{array}$ & $\mathbf{L R}_{1}$ & $\mathbf{E R}_{1}$ & $\begin{array}{l}U_{1} \text { in } \\
\text { mill. } €\end{array}$ & $\begin{array}{l}I_{1} \text { in } \\
\text { mill. } €\end{array}$ & $\begin{array}{l}\mathrm{E}_{1} \text { in } \\
\text { mill. } €\end{array}$ \\
\hline 1 & 75 & -31.75 & $-26.93 \%$ & $-6.78 \%$ & $-14.84 \%$ & 245.14 & 0.03 & $122.58 \%$ & $11.13 \%$ & -65.46 & -38.18 & -103.64 \\
\hline 2 & 75 & -21.98 & $-39.81 \%$ & $4.26 \%$ & $-13.36 \%$ & 239.14 & 0.45 & $119.79 \%$ & $10.99 \%$ & -59.77 & -34.38 & -94.16 \\
\hline 3 & 75 & -16.84 & $-43.55 \%$ & $3.40 \%$ & $-15.38 \%$ & 229.52 & 0.10 & $114.81 \%$ & $10.74 \%$ & -49.61 & -39.56 & -89.17 \\
\hline 4 & 75 & -16.62 & $-19.74 \%$ & $-5.01 \%$ & $-10.90 \%$ & 239.72 & 0.96 & $120.34 \%$ & $11.02 \%$ & -60.89 & -28.05 & -88.95 \\
\hline 5 & 75 & -15.38 & $-22.56 \%$ & $0.57 \%$ & $-8.69 \%$ & 245.09 & 0.02 & $122.55 \%$ & $11.13 \%$ & -65.40 & -22.35 & -87.75 \\
\hline 6 & 75 & -15.35 & $-15.53 \%$ & $8.80 \%$ & $-0.93 \%$ & 264.25 & 0.40 & $132.32 \%$ & $11.62 \%$ & -85.32 & -2.40 & -87.72 \\
\hline 8 & 75 & -12.99 & $-23.38 \%$ & $-1.98 \%$ & $-10.54 \%$ & 237.67 & 0.47 & $119.07 \%$ & $10.95 \%$ & -58.30 & -27.13 & -85.43 \\
\hline 9 & 75 & -12.03 & $-17.60 \%$ & $-2.83 \%$ & $-8.74 \%$ & 240.30 & 1.49 & $120.89 \%$ & $11.04 \%$ & -62.02 & -22.48 & -84.49 \\
\hline 10 & 75 & -11.33 & $-29.89 \%$ & $-2.19 \%$ & $-13.27 \%$ & 228.86 & 0.82 & $114.84 \%$ & $10.74 \%$ & -49.68 & -34.13 & -83.81 \\
\hline 11 & 75 & -11.01 & $-39.02 \%$ & $-1.76 \%$ & $-16.66 \%$ & 220.36 & 0.45 & $110.40 \%$ & $10.52 \%$ & -40.63 & -42.87 & -83.50 \\
\hline 12 & 75 & -10.81 & $-3.01 \%$ & $5.99 \%$ & $2.39 \%$ & 268.67 & 0.03 & $134.35 \%$ & $11.72 \%$ & -89.45 & 6.15 & -83.31 \\
\hline 13 & 75 & -10.00 & $-50.09 \%$ & $-3.46 \%$ & $-22.11 \%$ & 204.40 & 1.71 & $103.06 \%$ & $10.15 \%$ & -25.65 & -56.88 & -82.53 \\
\hline 14 & 75 & -9.86 & $-22.84 \%$ & $4.35 \%$ & $-6.53 \%$ & 244.29 & 1.00 & $122.65 \%$ & $11.13 \%$ & -65.59 & -16.80 & -82.38 \\
\hline
\end{tabular}

$\mathrm{EC}_{0}$ : equity capital at the end of period $0 ; \mathrm{EC}_{1}$ : equity capital at the end of period $1 ; \mathrm{r}_{11}$ : high-risk investment return in period $1 ; \mathrm{r}_{21}$ : low-risk investment return in period $1 ; \mathrm{r}_{\mathrm{p} 1}$ : return of the investment portfolio in period $1 ; \mathrm{C}_{\mathrm{ncat}, 1}$ : non-catastrophic claims in period $1 ; \mathrm{C}_{\mathrm{cat}, 1}$ : catastrophic claims in period $1 ; \mathrm{LR}_{1}$ : loss ratio in period $1 ; \mathrm{ER}_{1}$ : expense ratio in period $1 ; \mathrm{U}_{1}$ : underwriting result in period $1 ; \mathrm{I}_{1}$ : investment result in period $1 ; \mathrm{E}_{1}$ : earning in period 1 .

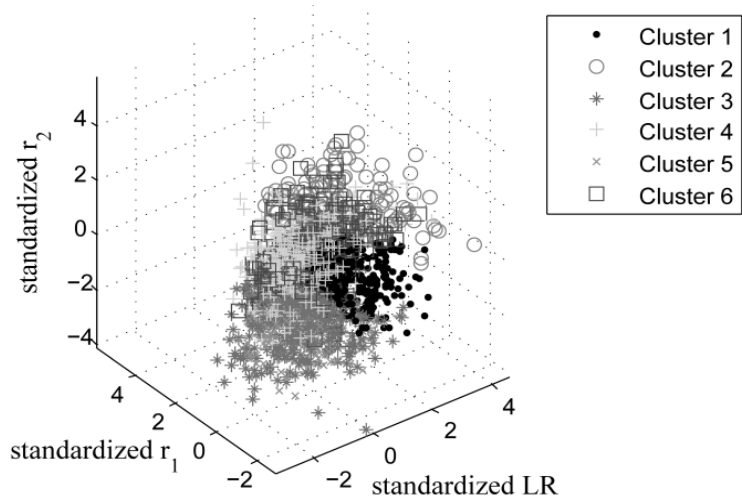

$\mathrm{r}_{\mathrm{p}}$ : return of the investment portfolio; LR: loss ratio.

Figure 1. 3D plot of the clustered risk factors of the 1,771 worst scenarios assuming independence.
Finally, for all poor scenarios across all dependence structures - not only for the case of the 15 worst scenarios without correlation modeling - very adverse trends in the capital market and/or very unfavorable claim developments are responsible for the insurer's ruin. The loss ratio is, without exception, well above the simulation average. It is different for investment returns; in this case, the entire range of developments (from good to normal to bad) is covered.

To analyzing these poor scenarios a cluster analysis using the Ward's minimum variance method [32] has been done. At the beginning of the clustering process, the following variables are selected for clustering because they have been identified as relevant characteristics: the loss ratio, high-risk investment return and low-risk investment return. For each of the eight dependence structures, the worst scenarios are divided into six clusters, in consideration of different 
population sizes, and the corresponding mean values of the risk factors and financial values are calculated. The clustering of the poor scenarios under the assumption of independence is shown in Figure 1. The figure shows a three-dimensional representation of the clustering variables, with one characteristic plotted on each axis. The three variables are standardized so that they can be directly compared to one another and plotted in an unbiased fashion. Detailed results of the clustering procedure are available upon request.

In addition, the clusters can be assigned to the case groups presented in Table 4 to compare the results for the different dependencies. Case 1 contains scenarios that are characterized by very negative trends on the capital market for both high-risk and low-risk investment returns; an increased loss ratio therefore cannot be compensated. Case 1' represents an attenuation of case 1 with respect to the investment returns. Case 2 differs from case 1 in the breakdown of the investment results. The high-risk investments result in such poor investment performance that even an acceptable low-risk investment return cannot substantially improve the performance of the company. The high-risk investment return in case $2^{\prime}$ is not as bad as that in case 2; however, this improvement in the high-risk investment return coincides with a change for the worse in the low-risk investment return and loss ratio. In case 3, those scenarios that exhibit an extreme loss ratio in addition to very poor investment returns are summarized. Case 3' aims to improve those scenarios, in which the extreme loss ratio is the decisive factor for the ruin, not those scenarios that display unfavorable trends on the capital market.

Table 4. Case groups of the clustering variables.

\begin{tabular}{|c|c|c|c|c|c|c|}
\hline Case & 1 & $1^{\prime}$ & 2 & $2^{\prime}$ & 3 & $3^{\prime}$ \\
\hline $\mathrm{r}_{1}$ & extremely negative & negative & extremely negative & negative & strongly negative & normally as expected \\
\hline $\mathrm{r}_{2}$ & extremely negative & negative & positive & closed to null & strongly negative & normally as expected \\
\hline LR & increased & increased & increased & high & extreme & extreme \\
\hline
\end{tabular}

$\mathrm{r}_{1}$ : high-risk investment return; $\mathrm{r}_{2}$ : low-risk investment return; LR: loss ratio.

The categorization of the poor scenarios into the six case groups (exemplary without correlation, using the Gauss and the Clayton copula) and the corresponding case mean values of the risk factors are shown in Figure 2. If only the 100 worst scenarios are taken into account in the case without correlation, then approximately $40 \%$ of the scenarios can be attributed to the cases characterized by extremely negative returns for both investments (see cases 1 and 1') and to the cases in which the high-risk investment returns resulted in a large loss (see cases 2 and 2'). Furthermore, the extreme loss ratio is the deciding factor (see case $3^{\prime}$ ) in $16 \%$ of the scenarios. The distribution over the case groups is more balanced when a higher number of scenarios is included. In particular, the increase in case 3 ', characterized primarily by a high loss ratio, is accompanied by a decrease in the cases of extremely negatively high-risk investment returns (see cases 1 and $2^{\prime}$ ). Case 3 , with very negative trends across all three risk factors, does not occur because no correlations between the risk factors are modeled.

The case group distribution when the Gauss copula is used is shown in the middle of Figure 2. Of the 100 worst scenarios, $84 \%$ of the scenarios correspond to case 1 and $16 \%$ correspond to case 3 . The other cases do not occur at all. Once again, the proportions change depending on the number of considered scenarios. The distribution is more adjusted to the independent case when a higher number of scenarios are taken into the consideration. The frequency with which the case groups are represented differs from that with no correlation owing to the linear dependencies included in the Gauss copula. Therefore, unfavorable developments of all of the risk factors are more often due to the choice of the correlations. The poor scenarios characterized by high loss ratios dominate when considering a large data base.
Extremely negative investment returns clearly gain significance when a reduced data base is considered.

This tendency is still clearly recognizable when the lower tail dependence Clayton copula is used (see Figure 2, right hand panel). Almost all of the worst possible scenarios are attributed to case 1 (94\% among the 100 worst scenarios and $76 \%$ among the 500 worst scenarios). The number of scenarios that are attributed to the other cases is higher when a larger number of poor scenarios are considered implying a gradual decrease in the occurrence of case 1 ; however, case 1 remains dominant.

An overview of the risk factors (return of the investment portfolio, loss ratio) corresponding to case group 1 for all of the dependence structures is shown in Figure 3. In the left diagram, the factors are the means of the scenarios corresponding to case 1 for the data base, including the 100 worst scenarios. The loss ratio is plotted on the $\mathrm{x}$-axis, and the portfolio return is plotted on the y-axis. The portfolio return is calculated by weighting the returns of the high-risk and low-risk investments. The loss ratio is above $105 \%$ for all of the dependency structures, and the return of the investment portfolio is below $-13 \%$ due to the extremely negative returns of the high-risk and low-risk investments. A loss ratio of $110.74 \%$ and a portfolio return of $-13.66 \%$ are obtained when independence is assumed. The stress levels of the individual balance sheet items are strongly affected by the correlation assumptions. Depending on the copula type, the loss ratio changes by $-4.43 \%$ (from $110.74 \%$ to $105.83 \%$ using the survival Clayton copula) to $10.80 \%$ (from $110.74 \%$ to $122.70 \%$ using the survival Gauss copula) and the portfolio return decreases by $7.65 \%$ (from $-13.66 \%$ to -14.71 using the Frank copula) up to $76.06 \%$ (from $-13.66 \%$ to $24.05 \%$ using the survival Gumbel copula). In the stress 
situation, the indication employing lower tail dependence copulas ( $t$, Clayton and survival Gumbel copula) is significantly stronger than the indication employing copulas exhibiting no tail dependence (Gauss and Frank copula) and upper tail dependence copulas (Gumbel and survival Clayton copula).

The diagram on the right hand side of Figure 3 presents an overview of the risk factors corresponding to case group 1 for a specified set of the 100,500 or 2,500 worst scenarios, from right to left in the diagram. When the 100 (first point from the right for each different type of dependency structure), 500 (second point from the right) or 2,500 (third point from the right) worst scenarios are considered, the following two effects are apparent. On the one hand, the forms of the stress scenarios are reduced for an increasing number of considered scenarios. On the other hand, the forms of the stress scenarios for different dependence structures become increasingly uniform when the number of considered scenarios increases. Note that in the cases using the upper tail dependence Gumbel or survival Clayton copula, case 1 does not occur in a set of the 2,500 worst scenarios. The reason for this observation is that the cluster within the set of 2,500 scenarios is attributed to the softer case 1 ' because its attributes are less pronounced compared to its attributes within a smaller set of scenarios.

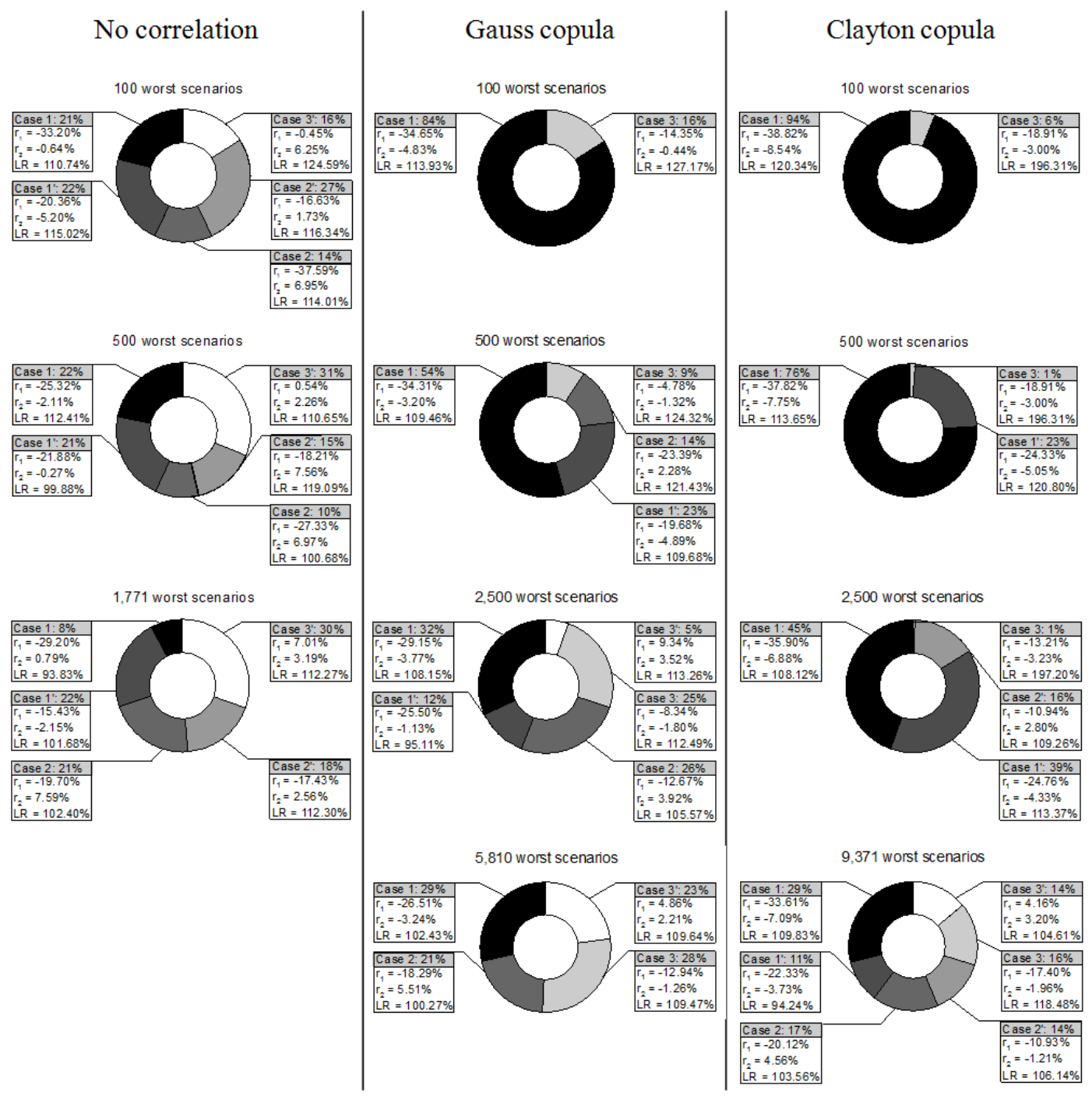

$r_{1}$ : high-risk investment return; $r_{2}$ : low-risk investment return; LR: loss ratio.

Figure 2. Distributions of the case groups and corresponding means of the risk factors. 

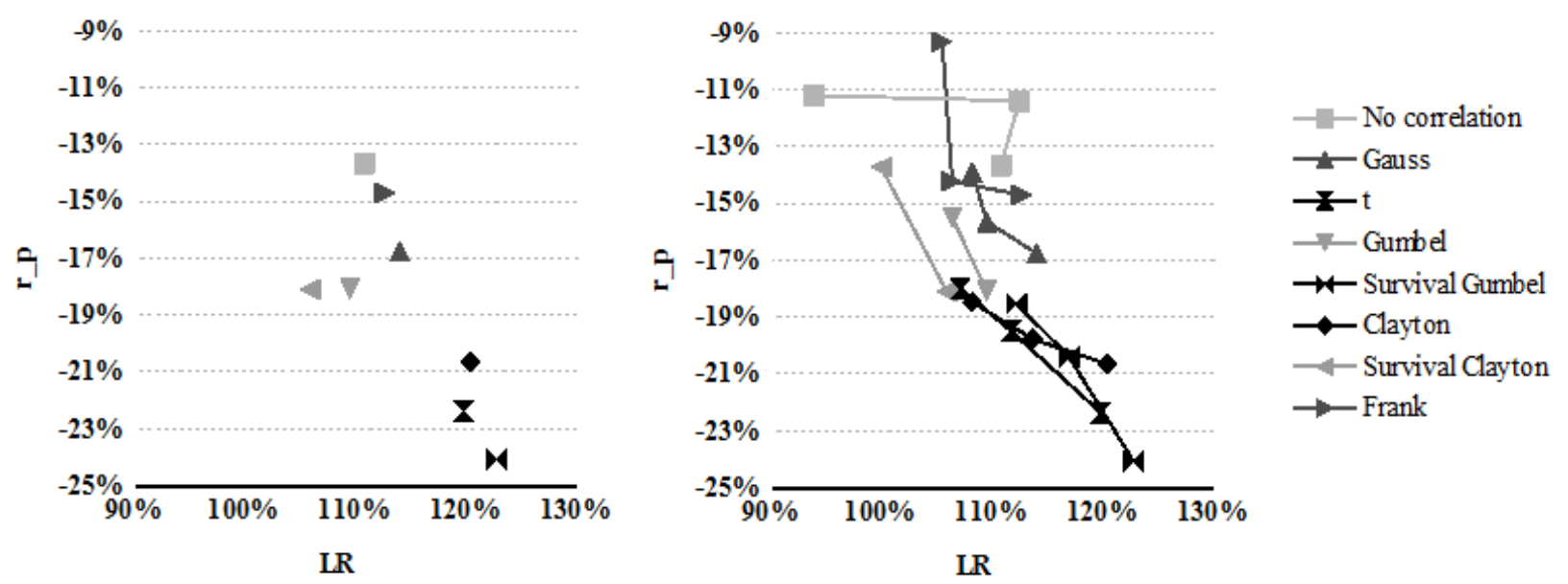

$\mathrm{r}_{\mathrm{p}}$ : return of the investment portfolio; LR: loss ratio.

Figure 3. General survey of the risk factors for case group 1.

The left side of Figure 4 contains an overview of the risk factors corresponding to case group 2 , based on a population of the 100 worst scenarios, for all of the different dependency structures. Case 2 is not represented in the case of the Gauss, survival Gumbel, Clayton or t copula, primarily due to the fact that the scenarios in which lower tail dependence is modeled are predominantly assigned to case 1 because of the simultaneous extreme unfavorable attributes of the risk factors. In the case of the Gauss copula, in which the (random) risk factors are linearly dependent, negative influences (as well as other influences) are strengthened. The comparison of the remaining dependence structures shows relatively small impacts on the loss ratio and portfolio return. In the case of independence, the loss ratio is $114.01 \%$ and the portfolio return is $-10.87 \%$. The loss ratio increases from $0.84 \%$ to $4.26 \%$, depending on the copula type, while the portfolio return changes by $-6.92 \%$ to $5.61 \%$.
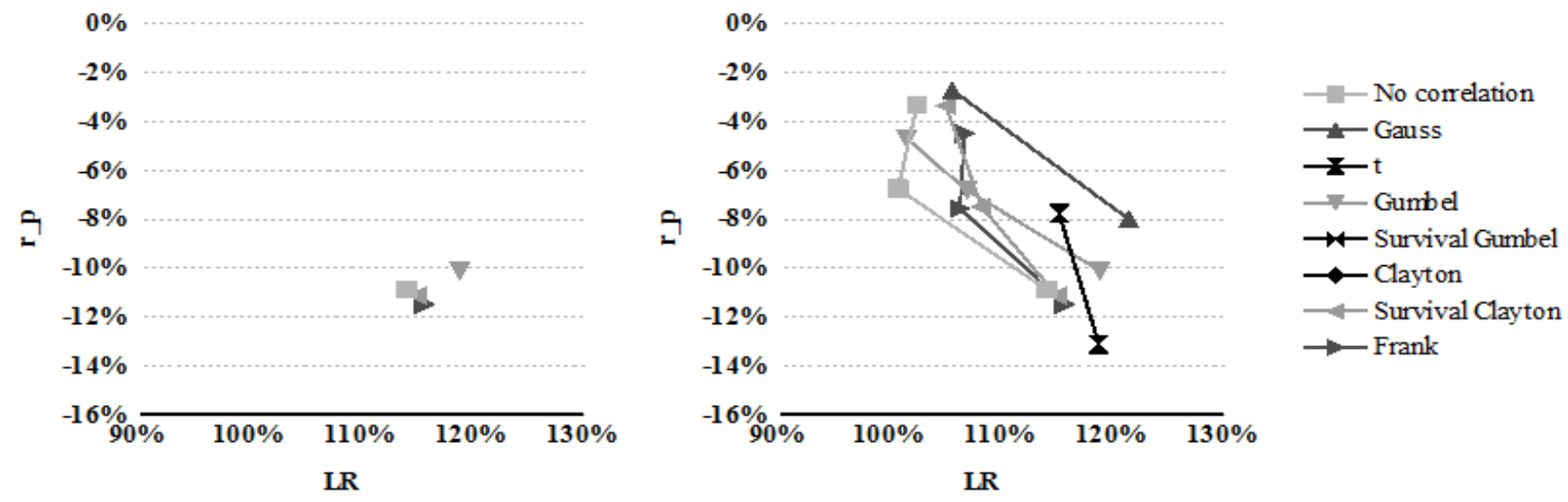

$r_{p}$ : return of the investment portfolio; LR: loss ratio.

Figure 4. General survey of the risk factors for case group 2.

When the analysis accounts for the 500 or 2,500 worst scenarios (see Figure 4, right hand panel) the forms of the stress scenarios within a given dependence structure and the differences in the forms of the stress scenarios between different dependence structures weaken. However, the additional points from the $t$ and Gauss copula deviate slightly from the overall picture, and case 2 is still not represented when using the survival Gumbel or the Clayton copula.

The overview is omitted for the case groups 1' and 2' because these overviews lead no new significant findings. In these cases, both the indication in the stress situation and the weakening of the risk factors are smaller than those in cases 1 and 2 due to the class definition.
The overview of the risk factors for case group 3 is shown in Figure 5. The simultaneous occurrence of a very negative investment portfolio and a very high loss ratio is a characteristic of this case. This case can be represented only by lower tail dependence copulas and the Gauss copula because the lower tail dependence copulas can account for the dependencies that occur during extreme events. The risk factors display a large variation between the different copulas. For example, for the worst 100 scenarios, the survival Gumbel copula leads to a loss ratio of $181.99 \%$ and a portfolio return of $-26.65 \%$, whereas the loss ratio is $126.91 \%$ and the portfolio return is $-16.18 \%$ for the $t$ copula. Moreover, the differences remain even when a larger number of worst 
scenarios is considered because the number of scenarios attributed to case 3 is quite small, which leads to a high variability between different dependence structures.
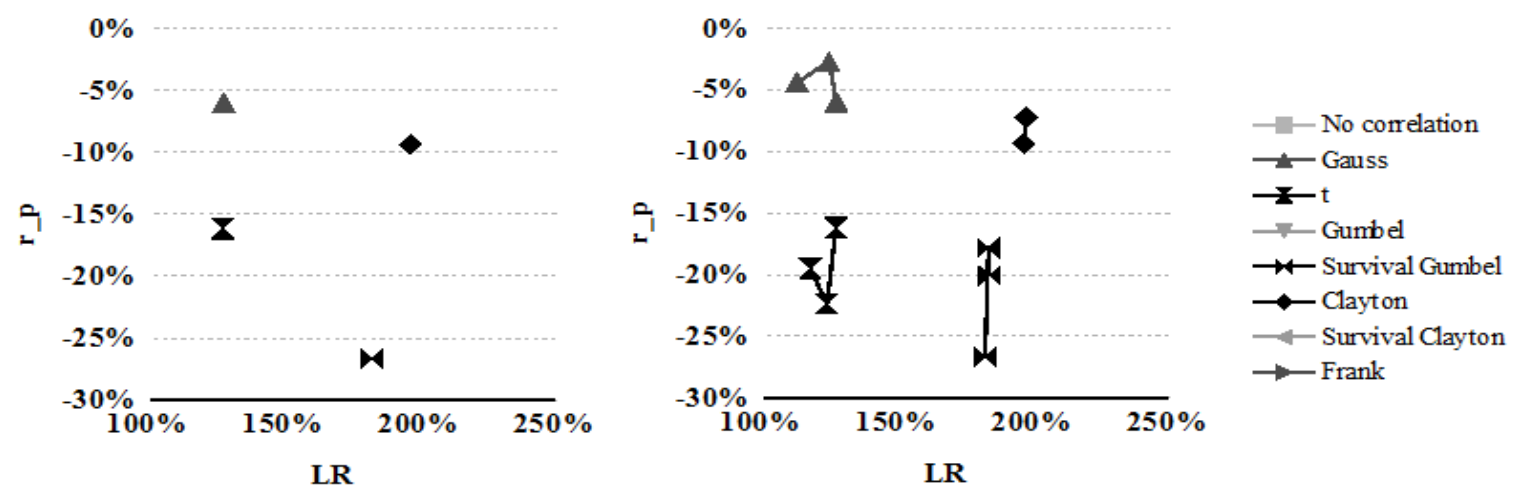

$\mathrm{r}_{\mathrm{p}}$ : return of the investment portfolio; LR: loss ratio.

Figure 5. General survey of the risk factors for case group 3.

Figure 6 illustrates the risk factors for case group 3' for the various correlation assumptions. The returns of the investment portfolio are generally positive (with one exception) but typically fall below the expected value of $7 \%$. The range of loss ratios reaches nearly $100 \%$. In particular, for the 100 worst scenarios, the loss ratio is extremely high under the lower tail dependence survival Gumbel copula $(181.61 \%)$ and upper tail dependence survival Clayton copula $(190.52 \%)$. Increasing the basis enables the loss ratios for the various dependence structures to converge relatively quickly and thoroughly, and the extreme deviations disappear.
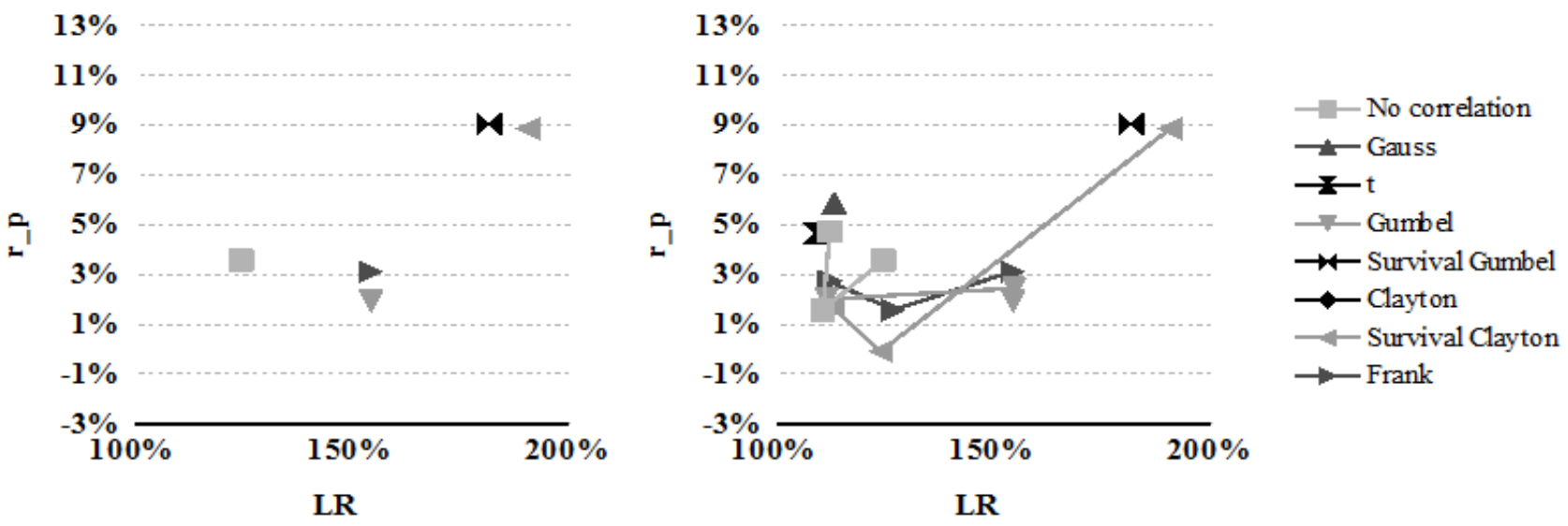

$r_{p}$ : return of the investment portfolio; LR: loss ratio.

Figure 6. General survey of the risk factors for case group 3'.

In the approach taken in this paper, the six clusters for each dependence structure and the considered set of poor scenarios are defined to be the stress scenarios. The stress scenarios can be very different in the form and structure of the risk factors. Finally, note that the true worst case scenarios are obtained under the assumption of lower tail dependence, for example, using the Clayton copula. The deduction of the correlation should be undertaken with the aim of obtaining a worst case scenario because the limited data available to insurance companies render the determination of the dependence difficult.

\section{Conclusions}

In this paper, the development of stress scenarios for stress testing using DFA modeling is investigated. Selected poor scenarios, which arise when the dependence structure is modeled using various copulas, are considered. This approach accounts for the possible correlations between the various assets and liabilities in the stress case. This study focuses on eight different implementations of the dependency relationship.

The analysis leads to three main findings, each concerning the risk management procedures of the insurance company. First, a systematic method is introduced for generating stress scenarios specific to the company and current economic environment using a DFA model. The identified stress scenarios are plausible, rigorous and comprehensive because the company-specific model reflects the actual risk situation of the company. In practice, the construction of simulation models with adequate functionality in the boundary areas is an important challenge. That particularly means the correct 
modeling of the maximum loss for each individual risk position as well as the maximum loss for the entire risk profile in consideration of non-linear dependencies. If a DFA model is available, then the new method does not require any additional resources, leading to widespread practical implementation.

Second, as demonstrated by the study of the critical scenarios, non-linear dependencies strongly influence the severity of the stress. Lower tail dependence copulas such as the Clayton and $\mathrm{t}$ copulas provide significantly stronger indications in the stress situation, as several risk factors are affected simultaneously. Particularly in the derivation of extremely severe scenarios, the modeling of asymmetric nonlinear dependencies therefore appears to be relevant. This result is of special importance for regulators and rating agencies, as current company models typically model only linear correlations or non-linear dependencies employing the Gauss copula. In addition, the stress tests currently employed by insurers use scenarios related to selected individual risk factors rather than considering all of the risk factors that are related to the solvency of the insurer simultaneously.

Third, different quantities of poor scenarios are considered, and it turns out that the number of scenarios has a strong impact on the form and structure of the risk factors. For a given individual dependence structure, the stress scenarios within each case group exhibit a distinct weakening when the number of considered poor scenarios increases. Furthermore, the stress scenarios for a given case group clearly converge across different dependence structures.

The implications of these findings are multifaceted. The work of the staff department is immediately affected. This paper therefore presents an effective approach to systematic searching for objective worst case scenarios. The major problem with current stress tests is that they employ handpicked scenarios, which provide deceptive security. The results of these tests are often misinterpreted because the tests consider implausible scenarios rather than the scenarios that could actually threaten the existence of the company. The derivation of stress scenarios from DFA models avoids this problem. The risk management process should therefore be complemented by adverse outcomes scenarios determined using lower tail dependence copulas.

More broadly, the concepts covered in this paper can inform discussions on general business principals, such as the strategy of the management and the rewarding of the management for their successes. For example, negative consequences must be considered when intending large rewards for the realization of good scenarios. Based on game theory, the management designs its work to maximize the expected profit, that is, the product of the profit and its probability. This strategy increases the variance of the financial results and thereby increases the probability of high losses. This effect must be eliminated for the rewarding of the management which requires proving in a model that higher profits are not linked with increased stress scenarios.

\section{Acknowledgements}

The authors are grateful to Martin Eling, Denis Toplek, Hans-Joachim Zwiesler and the participant of the annual conference of the German Association of Insurance Sciences 2013, Berlin for valuable suggestions and comments.

\section{Appendix}

Table 5. Balance sheet ratios and market parameters of the application study.

\begin{tabular}{lll}
\hline Parameter & Symbol & Initial value at $\boldsymbol{t}=\mathbf{0}$ \\
\hline Equity capital at the end of period $t$ & $E C_{t}$ & $€ 75$ million \\
Portion invested in high-risk investments in period $t$ & $\alpha_{t-1}$ & 0.40 \\
Tax rate & $t r$ & 0.25 \\
Risk free return & $r_{f}$ & 0.03 \\
\hline
\end{tabular}

Table 6. Premium and costs of the application study.

\begin{tabular}{|c|c|c|}
\hline Parameter & Symbol & Initial value at $\mathbf{t}=\mathbf{0}$ \\
\hline Premium income in period $t$ & $P_{t}$ & $€ 200$ million \\
\hline Underwriting market volume & $M V$ & $€ 1,000$ million \\
\hline Market growth & $i$ & 0.03 \\
\hline Company's underwriting market share in period $t$ & $\beta_{t-1}$ & 0.02 \\
\hline Premium rate level in period $t$ & $\Pi_{t}$ & 1 \\
\hline Autoregressive process parameter for lag 0 & $\mathrm{a}_{0}$ & 1.191 \\
\hline Autoregressive process parameter for lag 1 & $\mathrm{a}_{1}$ & 0.879 \\
\hline Autoregressive process parameter for lag 2 & $\mathrm{a}_{2}$ & -0.406 \\
\hline Consumer response function & $c r_{t-1}$ & 1 \\
\hline Upfront expenses linearly depending on the written market volume & $\gamma$ & 0.05 \\
\hline Claim settlement costs as portion of claims & $\delta$ & 0.05 \\
\hline
\end{tabular}


Table 7. Claim and return distributions of the application study.

\begin{tabular}{|c|c|c|}
\hline Parameter & Symbol & Initial value at $t=0$ \\
\hline Log-normal non-catastrophic claims as portion underwriting market share & $C_{\text {ncat }}$ & \\
\hline Mean claims & $E\left(C_{\text {ncat }}\right)$ & $€ 170$ million \\
\hline Standard deviation of claims & $\sigma\left(C_{n c a t}\right)$ & $€ 17$ million \\
\hline Pareto distributed catastrophic claims & $C_{c a t}$ & \\
\hline Mean claims & $E\left(C_{\text {ncat }}\right)$ & $€ 0.5$ million \\
\hline Dispersion parameter & $D\left(C_{\text {ncat }}\right)$ & 4.5 \\
\hline Normally distributed high-risk investment return in period $t$ & $r_{1 t}$ & \\
\hline Standard deviation of return & $\sigma\left(r_{1 t}\right)$ & 0.2 \\
\hline Normally distributed low-risk investment return in period $t$ & $r_{2 t}$ & \\
\hline Mean return & $E\left(r_{2 t}\right)$ & 0.05 \\
\hline Standard deviation of return & $\sigma\left(r_{2 t}\right)$ & 0.05 \\
\hline
\end{tabular}

Table 8. Correlations of the application study.

\begin{tabular}{lll}
\hline Parameter & Symbol & Initial value at $\boldsymbol{t}=\mathbf{0}$ \\
\hline Kendall's rank correlation between high-risk and low-risk investments & $\rho_{\tau 1}$ & 0.2 \\
Kendall's rank correlation between non-catastrophic and catastrophic losses & $\rho_{\tau 2}$ & 0.2 \\
Kendall's rank correlation between assets and liabilities & $\rho_{\tau 1}$ & -0.1 \\
\hline
\end{tabular}

Table 9. Dependency properties of the considered elliptical copulas [18].

\begin{tabular}{lllll}
\hline Copula & Tail Dependence & Kendall's Tau $\rho_{\tau}$ & Parameter & $\lambda_{u}$ \\
\hline Gauss & none & $\frac{2}{\pi} \arcsin (\rho)$ & $|\rho| \leq 1$ & 0 \\
& & 2 & & $\lambda_{l}$ \\
$\mathrm{t}_{v}$ & upper and lower & $\frac{2}{\pi} \arcsin (\rho)$ & $|\rho| \leq 1$ & $2 \bar{t}_{v+1}\left(\sqrt{v+1} \frac{\sqrt{1-\rho}}{\sqrt{1+\rho}}\right)$ \\
\hline
\end{tabular}

Table 10. Dependency properties of the considered hierarchical Archimedean copulas (HACs) with completely montone generators and corresponding parameter ranges [18,37].

\begin{tabular}{|c|c|c|c|c|c|c|c|}
\hline Copula & $\begin{array}{l}\text { Tail } \\
\text { Dependence }\end{array}$ & Generator $\phi_{i}(u)$ & Kendall's Tau $\rho_{\tau i}$ & & $\begin{array}{l}\text { Parameter, } \\
\left(\phi_{1}^{-1} \circ \phi_{2}^{-1}\right)^{\prime} \text { c.m. }\end{array}$ & $\lambda_{u}$ & $\lambda_{l}$ \\
\hline Gumbel & upper & $\exp \left\{-u^{1 / \theta_{i}}\right\}$ & $1-\frac{1}{\theta_{i}}$ & & $\theta_{1} \leq \theta_{2}, \theta_{i} \in[1, \infty)$ & $2-2^{\frac{1}{\theta_{i}}}$ & 0 \\
\hline $\begin{array}{l}\text { Survival } \\
\text { Gumbel }\end{array}$ & lower & via Gumbel & $1-\frac{1}{\theta_{i}}$ & & $\theta_{1} \leq \theta_{2}, \theta_{i} \in[1, \infty)$ & 0 & $2-2^{\frac{1}{\theta_{i}}}$ \\
\hline Clayton & lower & $\left(\theta_{i} u+1\right)^{-1 / \theta_{i}}$ & $\frac{\theta_{i}}{\theta_{i}+2}$ & & $\theta_{1} \leq \theta_{2}, \theta_{i} \in(0, \infty)$ & 0 & $2^{-\frac{1}{\theta_{i}}}$ \\
\hline $\begin{array}{l}\text { Survival } \\
\text { Clayton }\end{array}$ & upper & via Clayton & $\frac{\theta_{i}}{\theta_{i}+2}$ & & $\theta_{1} \leq \theta_{2}, \theta_{i} \in(0, \infty)$ & $2^{-\frac{1}{\theta_{i}}}$ & 0 \\
\hline Frank & none & $-\frac{1}{\theta_{i}} \ln \left\{e^{-u}\left(e^{-\theta_{i}}-1\right)+1\right\}$ & $1-4 \theta_{i}^{-1}\left(1-\frac{1}{\theta_{i}} \int_{0}^{\theta}\right.$ & $\left.\frac{t}{\exp (t)-1} d t\right)$ & $\theta_{1} \leq \theta_{2}, \theta_{i} \in(0, \infty)$ & 0 & 0 \\
\hline
\end{tabular}

\section{References}

[1] J. Berkowitz, "A coherent framework for stress testing", Journal of Risk, vol. 2, no. 2, 2000, pp. 1-11.

[2] BaFin, Rundschreiben 1/2004 (VA) - Durchführung von Stresstests, Bonn: Bundesanstalt für Finanzdienstleistungsaufsicht, February 2004.

[3] FSA, Stress and scenario testing (CP 08/24), London: Financial Services Authority, 2008.

[4] BCBS, Amendment to the capital accord to incorporate market risk, Basel: Basel Committee on Banking Supervision, November 2005.

[5] G. Bonti, M. Kalkbrener, C. Lotz, and G. Stahl, "Credit risk concentrations under stress", Journal of Credit Risk, vol. 2, no. 3, 2006, pp. 115-136.
[6] ECB, Financial stability report, Frankfurt a. M.: European Central Bank, June 2006.

[7] H.-J. Zwiesler, "Asset-Liability-Management - die Versicherung auf dem Weg von der Planungsrechnung zum Risikomanagement", in: K. Spremann (ed.), Versicherungen im Umbruch, Berlin: Springer, 2005, pp. 117-131.

[8] P. M. Achleitner, J. Biebel, and D. Wichels, "Does WTC matter for the investment policy of $\mathrm{p} / \mathrm{c}$ insurance companies?", The Geneva Papers on Risk and Insurance, vol. 27, no. 2, 2002, pp. 275-282.

[9] CAS, DFA Handbook, Arlington: Casualty Actuarial Society, June 1999.

[10] S. P. Lowe, and J. N. Stanard, "An integrated dynamic financial analysis and decision support system for a property catastrophe reinsurer", ASTIN Bulletin, vol. 27, no. 2, 1997, pp. 339-371. 
[11] R. Kaufmann, A. Gadmer, and R. Klett, "Introduction to dynamic financial analysis", ASTIN Bulletin, vol. 31, no. 1, 2001, 213-249.

[12] P. Blum, M. Dacorogna, P. Embrechts, T. Neghaiwi, and H. Niggli, "Using DFA for modeling the impact of foreign exchange risks on reinsurance decisions", Casualty Actuarial Society Forum, 2001, pp. 49-93.

[13] S. P. D'Arcy, and R. Gorvett, "The use of dynamic financial analysis to determine whether an optimal growth rate exists for a property-liability insurer", Journal of Risk and Insurance, vol. 71, no. 4, 2004, pp. 583-615.

[14] M. Eling, T. Parnitzke, T., and H. Schmeiser, "Management strategies and dynamic financial analysis", Variance, vol. 2, no. 1, 2008, pp. 52-70.

[15] A. Sklar, "Fonctions de répartition à $\mathrm{n}$ dimensions et leurs marges", Publ. Inst. Statist. Univ. Paris, vol. 8, 1959, pp. 229231.

[16] S. Wang, "Aggregation of correlated risk portfolios: models and algorithms", Proceedings of the Casualty Actuarial Society, vol. 85 , no. 163 , 1998, pp. 848-939.

[17] E. W. Frees, and E. A. Valdez, "Understanding relationships using copulas", North American Actuarial Journal, vol. 2, no. 1, 1998, pp. $1-25$

[18] P. Embrechts, F. Lindskog, and A. McNeil, "Modelling dependence with copulas and applications to risk management", in: S.T. Rachev (ed.), Handbook of Heavy Tailed Distributions in Finance, Amsterdam: Elsevier, 2001, pp. 329-384.

[19] Y. Malevergne, and D. Sornette, "Testing the Gaussian copula hypothesis for financial assets dependencies", Quantitative Finance, vol. 3, no. 4, 2003, pp. 231-250.

[20] E. Kole, K. Koedijk, and M. Verbeek, "Selecting copulas for risk management", Journal of Banking \& Finance, vol. 31, no. 8, 2007, pp. 2405-2423.

[21] A. Dias, and P. Embrechts, "Testing for structural changes in exchange rates' dependence beyond linear correlation", The European Journal of Finance, vol. 15, no. 7-8, 2009, pp. 619637.

[22] N. Whelan, "Sampling from Archimedean copulas", Quantitative Finance, vol. 4, no. 3, 2004, pp. 339-352.

[23] M. Hofert, "Sampling Archimedean copulas", Computational Statistics \& Data Analysis, vol. 52, no. 12, 2008, pp. 51635174.

[24] A. McNeil, "Sampling nested Archimedean copulas", Journal of Statistical Computation and Simulation, vol. 78, no. 6, 2008, pp. 567-581.

[25] M. Eling, and D. Toplek, "Modeling and management of nonlinear dependencies - copulas in dynamic financial analysis", Journal of Risk and Insurance, vol. 76, no. 3, 2009, pp. 651-681.

[26] D. Diers, "Management strategies in multi-year enterprise risk management", The Geneva Papers on Risk and Insurance, vol. 36, 2011, pp. 107-125.

[27] J. Han, and M. Kamber, Data Mining: Concepts and Techniques, San Francisco: Morgan Kaufmann, 2006.
[28] D. Wishart, "An algorithm for Hierarchical classifications", Biometrics, vol. 25, no. 1, 1969, pp. 165-170.

[29] R. M. Cormack, "A review of classification", Journal of the Royal Statistical Society, vol. 134, no. 3, 1971, pp. 321-367.

[30] M. R. Anderberg, Cluster Analysis or Applications, New York: Academic Press, 1973.

[31] D. Scheibler, and W. Schneider, "Monte Carlo tests of the accuracy of cluster analysis algorithm: a comparison of hierarchical and nonhierarchical methods", Multivariate Behavioral Research, vol. 20, no. 3, 1985, pp. 283-304.

[32] J. H. Ward, "Hierarchical grouping to optimize an objective function", Journal of the American Statistical Association, vol. 58, no. 301, 1963, pp. 236-244.

[33] G. N. Lance, and W. T. Williams, "A general theory of classificatory sorting strategies", Computer Journal, vol. 9, no. 4, 1967, pp. 373-380.

[34] P. Mangiameli, S. K. Chen, and D. West, "A comparison of SOM neural network and hierarchical clustering methods", European Journal of Operational Research, vol. 93, no. 2, 1996, pp. 402-417.

[35] P. Embrechts, A. McNeil, and D. Straumann, "Correlation and dependence in risk management: properties and pitfalls", in: M. A. H. Dempster (ed.), Risk Management: Value at Risk and Beyond, Cambridge: Cambridge University Press, 2002, pp. 176-223.

[36] C. Hering, M. Hofert, J.-F. Mai, and M. Scherer, "Constructing hierarchical Archimedean copulas with Lévy subordinators", Journal of Multivariate Analysis, vol. 101, no. 6, 2010, pp. 1428-1433.

[37] R. B. Nelsen, An Introduction to Copulas, New York: Springer, 2006.

[38] G. Fusai, and A. Roncoroni, Implementing Models in Quantitative Finance: Methods and Cases, Berlin Heidelberg: Springer, 2008.

[39] C. Hering, M. Hofert, "Goodness-of-fit tests for Archimedean copulas in high dimensions", in: K. Glau, M. Scherer, and R. Zagst (ed.), Innovations in Quantitative Risk Management, Springer, 2013, pp. 357-373.

[40] O. Horn, and H.-J. Zwiesler, "Die Problematik der Anwendung von Managementregeln im Risikomanagement", in D. Zietsch (ed.), Beiträge zu aktuellen Themen des Versicherungsmarktes, Karlsruhe: Verlag Versicherungswirtschaft, 2008, pp. 79-125.

[41] W.-R. Heilmann, Fundamentals of Risk Theory, Karlsruhe: Verlag Versicherungswirtschaft, 1988.

[42] P. Artzner, F. Delbaen, J.-M. Eber, and D. Heath, "Coherent measures of risk", Mathematical Finance, vol. 9, 1999, pp. 203-228.

[43] P. Albrecht, Grundprinzipien der Finanz- und Versicherungsmathematik, Stuttgart: Schäffer-Poeschel Verlag, 2007.

[44] W. F. Sharpe, "Mutual fund performance", Journal of Business, vol. 39, no. 1, 1966, pp. 119-138. 